\title{
Novel flow apparatus for investigating shear-enhanced crystallization and structure development in semicrystalline polymers
}

\author{
Guruswamy Kumaraswamy, Ravi K. Verma, and Julia A. Kornfield ${ }^{\text {a) }}$ \\ Division of Chemistry and Chemical Engineering, California Institute of Technology, Pasadena, \\ California 91125
}

(Received 6 October 1998; accepted for publication 12 January 1999)

\begin{abstract}
An instrument to study the effects of shearing on the crystallization process in semicrystalline polymers is described. It can impose transient stresses similar to those encountered in polymer processing and provides in situ monitoring of microstructure development during and after cessation of flow. Box-like wall shear stress profiles (rise and fall times under $50 \mathrm{~ms}$ with maximum wall shear stress on the order of $0.1 \mathrm{MPa}$ ) can be applied for controlled durations. A unique feature of our device is that it accommodates a wide variety of real-time probes of structure such as visible and infrared polarimetry and light and x-ray scattering measurements. The design also allows us to retrieve the sample for ex situ optical and electron microscopy. Data are acquired with millisecond resolution enabling us to record the extent of shear deformation of the polymer melt during the pressure pulse. Our device works with small sample quantities (as little as $5 \mathrm{~g}$; each experiment takes $\sim 500 \mathrm{mg}$ ) as opposed to the kilogram quantities required by previous instruments capable of imposing comparable deformations. This orders-of-magnitude reduction in the sample size allows us to study model polymers and new developmental resins, both of which are typically available only in gram-scale quantities. The compact design of the shear cell makes it possible to transport it to synchrotron light sources for in situ x-ray scattering studies of the evolution of the crystalline structure. Thus, our device is a valuable new tool that can be used to evaluate the crystallization characteristics of resins with experimental compositions or molecular architectures when subjected to processing-like flow conditions. We demonstrate some of the features of this device by presenting selected results on isotactic polypropylenes. (C) 1999 American Institute of Physics.
\end{abstract}

[S0034-6748(99)04504-9]

\section{INTRODUCTION}

Macromolecules having chemical and structural regularity can, under certain conditions, arrange themselves into a semicrystalline state. Complete crystallization is kinetically frustrated and a significant amount of noncrystalline material is trapped, interspersed among the crystallites. A composite structure spontaneously forms, with the crystalline phase conferring strength and the noncrystalline fraction providing toughness and flexibility. The macroscopic properties of the material depend strongly on the fraction of crystalline material (called the degree of crystallinity) and on the morphology (the spatial arrangement, size and orientation distribution of the crystallites). The desirable balance of properties and the extent to which structure and material properties can be controlled through processing lead to a wide range of applications of semicrystalline polymers, including packaging and nonpackaging films, coatings, sheets, wires and cables and injection and blow molded objects. Worldwide use of polyolefins alone exceeded 125 billion pounds in $1997 .{ }^{1}$

The process of crystallization in polymer melts occurs by nucleation and growth; crystallites grow by reorganizing random-coil chains into chain-folded, platelet-like crystalline lamellae (typically $\sim 10 \mathrm{~nm}$ thick) separated by regions of

\footnotetext{
a) Author to whom correspondence should be addressed; electronic mail: jak@cheme.caltech.edu
}

noncrystalline material. During crystallization from a quiescent melt, the crystalline lamellae typically arrange to form sheaf-like stacks (consisting of a few lamellae, typically 50$100 \mathrm{~nm}$ thick). These stacks radiate outwards to form spherulites which can range in size from submicron to millimeters. ${ }^{2}$ The range of morphological length scales necessitates several measurement techniques for characterization.

The microstructure of a semicrystalline polymer depends strongly on processing history. ${ }^{3}$ Imposing flow fields on a crystallizing polymer melt can accelerate the rate of crystallization and result in the formation of oriented crystallites. ${ }^{4}$ Commercial polymer processing operations (such as extrusion, injection molding, film blowing or fiber spinning) subject a polymer melt to intense flow fields (shear, elongational or mixed) and crystallization occurs from the distorted melt. ${ }^{4,5}$ The effect of processing on semicrystalline structure is of interest because it has a profound effect on material properties, such as mechanical strength and gas permeability ${ }^{6-9}$ For example, the development of highly anisotropic crystallites during spinning gives certain polymeric aramide and polyethylene fibers a Young's modulus between 80 and $130 \mathrm{GPa}$ in the direction of orientation (compared to 1-3 GPa for typical semicrystalline polymers or $200 \mathrm{GPa}$ for steel). ${ }^{3}$ Thus, there is a strong motivation to observe structure development in real time during processing.

In spite of intensive research over the past three decades, the fundamental basis of the effects of processing on struc- 
ture development remains elusive. Processing alters the rate, form and anisotropy of crystallization. These effects are believed to arise through the interplay of flow and thermal history, melt relaxation dynamics and nucleation and growth kinetics. Three requirements for tackling such a complex problem are evident: the need to create a precisely controlled flow and thermal history, the need to monitor structure development in real time, and the need to perform experiments on materials with well-defined molecular structure. The first challenge is to generate a well-defined flow history with deformations that mimic processing conditions and to characterize the effects of flow while excluding those of temperature transients and gradients. This requirement has been addressed by Janeschitz-Kriegl and co-workers. ${ }^{10-13}$ The second requirement is that of monitoring structure development on scales that range from the segmental orientation distribution in the liquid state to the nanostructure of the crystalline lamellae and noncrystalline interlamellar material. To probe the effects of local molecular structure (e.g., stereoregularity or comonomer content) and macromolecular architecture (e.g., overall chain length, polydispersity or long-chain branching), well-defined materials with systematic control of these parameters should be studied. Such model polymers can be synthesized, but they are not available on a large scale. Therefore, instruments that can operate with modest amounts (a few grams) of a sample are required.

We have designed and constructed an instrument for observing the kinetics and morphology of crystallization in real time that meets all these criteria. In particular, it enables the application of well-defined intervals of shearing with shear stresses up to those typical of industrial processes with continuous monitoring of structural development during and after flow.

\section{BACKGROUND}

Given the obvious commercial relevance, various aspects of flow enhanced crystallization have been studied. These experiments can be broadly categorized into three types: (i) investigations that probe the effect of a complicated flow pattern particular to a specific processing geometry on the development of semicrystalline structure; (ii) investigations that use modified rheometers to generate better defined thermal and flow histories but which are typically unable to access processing-like conditions; (iii) studies that examine the effects of well controlled flow and thermal history that can access shear stresses comparable to processing conditions. ${ }^{10-13}$

Ex situ and in situ investigation ${ }^{8,9,14-27}$ of the effect of flows in "practical" geometries (e.g., extrusion, spinning) on structure development during crystallization has yielded considerable information on the effects of a particular type of processing flow on the resulting crystallization and material properties of the resin studied. While these experiments provide a direct link between practical processing parameters and structure development, the use of industrial processing equipment necessitates large quantities of sample and limits the range of conditions that can be applied and how well they can be controlled.
Well-defined flow histories can be applied by shearing the polymer using modified rheometers in sliding plate, torsional parallel disk, cone and plate or Couette geometries. ${ }^{28-38}$ Use of rheometers enabled experiments with small sample quantities under controlled isothermal conditions but, in most cases, the high levels of shear strain and strain rate typical of processing conditions could not be accessed. Further, in all such experiments (except the recent studies of Vleeshouwers and Meijer ${ }^{38}$ ) the subcooled polymer melt was subjected to continuous shear for the entire duration of the experiment. Thus, flow deformation is experienced by the melt as well as by the crystalline entities formed during the experiment. This causes a continuous change in the orientation distribution of the crystallites as they are formed and obscures the relation between the distortion of the melt and the final semicrystalline structure.

Elegant experiments by Janeschitz-Kriegl et al. ${ }^{10-13}$ clearly isolated the effects of flow from thermal effects and separated the effects of shear on anisotropic growth from the effects of shear on already crystallized material. Polymer held in a reservoir was injected into a slit die. The die, which was thermally isolated from the extruder, was held at a high temperature to erase the memory of the filling process and then cooled to the desired crystallization temperature (Fig. 1). Then the relaxed subcooled polymer melt was subjected to intense shearing for a brief "shearing time" (short enough that no detectable crystallization occurred). This experimental protocol generates well-defined initial conditions for the polymer melt and a controlled and simple deformation profile. The polymer was allowed to crystallize subsequently and the turbidity and birefringence were tracked to monitor the progress of crystallization. The crystallization temperatures were selected such that the shearing time was always much shorter than the crystallization time, thus minimizing the deformation of already formed crystallites. Since the high pressures used to drive the flow were generated by an extruder, large amounts of sample were required for operation of the instrument. This requirement severely limited the range of polymers that could be studied. Further, the rotating polarizer setup used for monitoring the birefringence limited the time resolution of their measurements making it difficult to monitor the deformation of the melt during short shearing times.

We have designed a shear device that retains the advantages of Janeschitz-Kriegl's apparatus while adding the capability to work with small amounts of sample. Further, a wide range of experimental probes has been incorporated that allows us to monitor chain conformation in the polymer melt during shear along with the development of the crystallite nanostructure and the semicrystalline microstructure during crystallization. Our device accommodates a variety of real-time, in situ probes of structure (optical and x-ray) and facilitates subsequent $e x$ situ characterization by optical and electron microscopies. Like the preceding instrument, our device generates a brief interval of shear by driving the polymer from a reservoir into a narrow slit. Data can be acquired with $5 \mathrm{~ms}$ time resolution during the pressure pulse to quantify the conformational distortion of polymers in the melt and relate it to the nature of the subsequent crystallization (viz. 


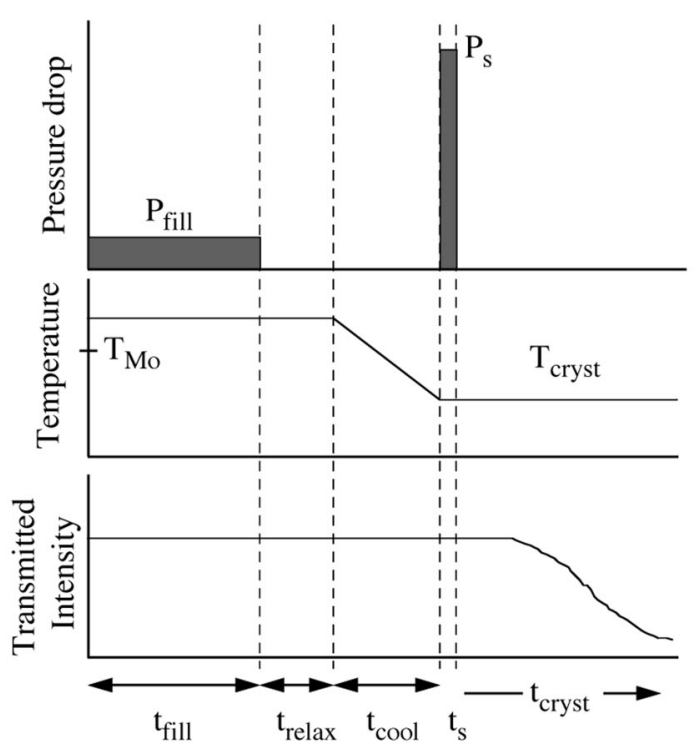

FIG. 1. Experimental protocol for shear-enhanced crystallization experiments. The polymer melt is extruded from the reservoir using a low pressure drop across the flow channel, $P_{\text {fill }}$, for a time $t_{\text {fill }}$ (top graph); then it is allowed to relax for time $t_{\text {relax }}$ at a temperature $T_{\text {melt }}$ that is above the equilibrium melting temperature $T_{\mathrm{Mo}}$ (middle graph). When the polymer melt has relaxed, it is cooled to the crystallization temperature, $T_{\text {cryst }}$ and then subjected to shear as it is extruded at a high pressure, $P_{s}$, for a brief interval, $t_{s}$. The progress of crystallization with time, $t_{\text {cryst }}$, is monitored using different probes (see the text), including turbidity (bottom graph).

the enhanced kinetics and development of anisotropic crystalline structures). Each experiment requires only about 500 $\mathrm{mg}$ of polymer. Recent advances in metallocene catalysis have made it possible to synthesize polyolefins with precisely defined molecular architectures and well controlled distribution of molecular weights. ${ }^{39}$ The modest sample requirement of our instrument enables studies of these welldefined, model polymers and of experimental resins that are available only in small quantities.

In Sec. III, we present a complete description of the design and operation of our instrument. We discuss the capabilities of the instrument and demonstrate our ability to impose precisely controlled wall shear stresses on the subcooled sample. We then present illustrative results on different isotactic polypropylene resins.

\section{INSTRUMENT DESIGN}

The design considerations behind our flow cell are driven by the experimental protocol established by Janeschitz-Kriegl and co-workers. ${ }^{10}$ The desired mode of operation is to fill the flow channel at low injection pressures and high temperatures $\left(>T_{M o}\right)$, hold at this temperature to erase melt memory effects, cool quickly to the desired crystallization temperature, apply a controlled wall shear stress, and monitor structure development during and after flow. An instrument that can perform this protocol is capable of doing a variety of other experiments, e.g., injecting a hot melt into a cold mold or applying continuous stress during crystallization. In addition, such an instrument could be used to observe transient flow-induced structure in other microstructured polymers, e.g., polymer blends, liquid crystalline polymers or block copolymers.
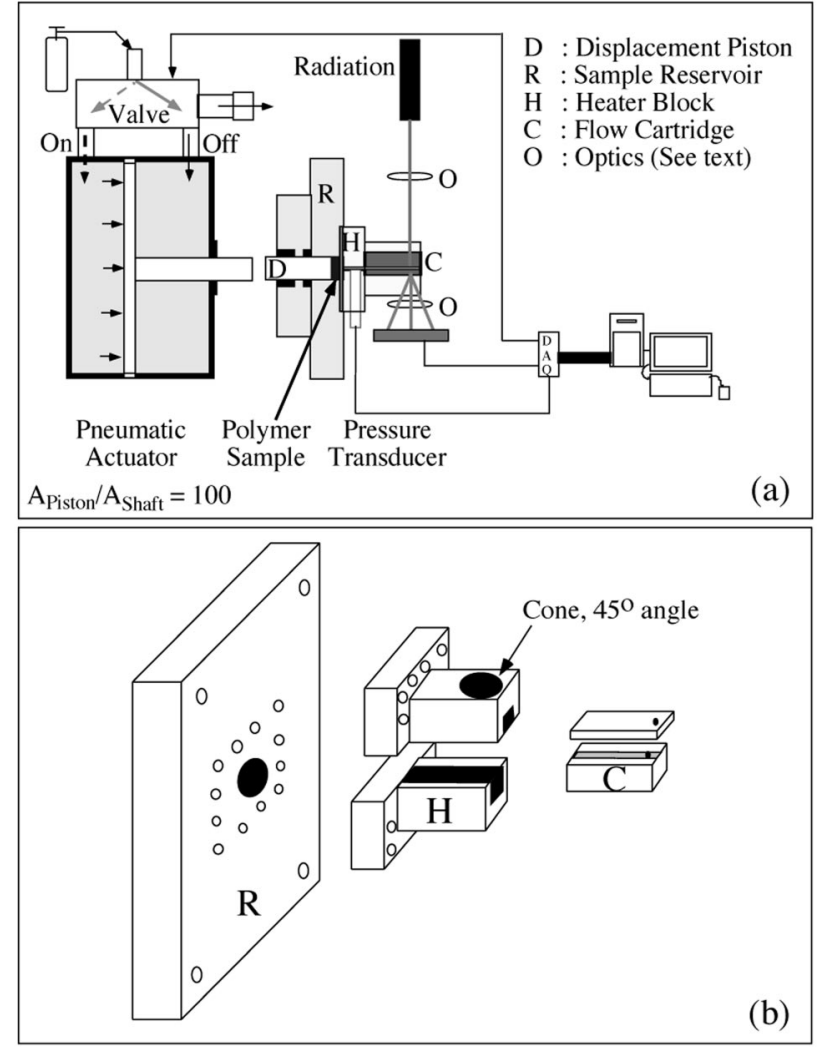

FIG. 2. (a) Schematic diagram of the shear instrument (see the text for details). Feedback controllers for temperature control and relays used to switch the valve are not shown. The recirculating system used for the heat transfer oil to keep the heater block isothermal is also not shown. (b) Isometric view of the reservoir $(\mathrm{R})$, heater block $(\mathrm{H})$ and cartridge $(\mathrm{C})$ indicating how they fit together. Note that $\mathrm{H}$ and $\mathrm{C}$ are machined in two parts that are assembled together. The reservoir block is mounted onto the pneumatic actuator using threaded rods (see the text) which pass through the holes indicated near the corners of $\mathrm{R}$. The heater block bolts onto the reservoir block as shown. The cartridge slides into $\mathrm{H}$ and is held in place by a plate that bolts onto the face of $\mathrm{H}$. The perspective shown in (b) is upside down relative to (a); the light exits through the conical aperture to permit light and $\mathrm{x}$-ray scattering. Details of the channels for circulating oil through the heater block, mounting holes for pressure transducer, heaters etc. have been omitted for clarity.

\section{A. Flow cell}

To study the effects of shearing time and stress on the subsequent crystallization behavior requires sharp deformation pulses (desired rise and fall times are less than $\sim 50 \mathrm{~ms}$ ), with control over the pulse duration (typically from $250 \mathrm{~ms}$ to $8 \mathrm{~s}$ ) and magnitude (wall shear stresses up to $\sim 0.1 \mathrm{MPa}$ ). These considerations necessitate a device based on pressure driven flow through a channel (we were unable to design a practical instrument that provides a uniform shear with the required specifications on rise and fall times). The flow channel is machined into an independent component called the "flow cartridge" [C in Figs. 2(a) and 2(b)] to facilitate removal of samples for ex situ analysis and also to provide flexibility in changing to a different flow geometry. Here we use a two-dimensional channel flow geometry because of its simplicity: a rectangular slit with an aspect ratio greater than 10 (width $=6.35 \mathrm{~mm}$, depth $=0.5 \mathrm{~mm}$ ) is cut into a stainless steel block. The length of the flow cartridge is $\sim 63.5 \mathrm{~mm}$ which limits the maximum shear strain to 120 . 
Other flow geometries can be easily accommodated by making an appropriate flow cartridge.

\section{B. Mechanical assembly}

The flow cartridge fits snugly in a large block which serves as a thermal reservoir [called the heater block, $\mathrm{H}$, in Figs. 2(a) and 2(b)]. The flow cartridge is held in place during experiments by a stainless steel restraining plate bolted onto the heater block. The restraining plate and the flow cartridge can be removed after experiments, enabling easy sample retrieval for ex situ microtoming and microscopy. The heater block is bolted onto an aluminum block in which a hole $(25.4 \mathrm{~mm}$ diam) serves as a melt reservoir $(\mathrm{R})$. The volume of the melt reservoir is significantly greater than that of the flow channel, thereby enabling several "fills" and experiments with a single loading of the melt reservoir.

The high pressure needed to extrude the polymer is transmitted to the melt in the reservoir through a displacement piston. The piston is a stainless steel rod ( $25 \mathrm{~mm}$ diam) equipped with a high temperature, high pressure lip seal (AS-92071, All Seals Inc., Santa Ana, CA). Pressures at the entrance of the flow channel are recorded through a pressure transducer (PT-462E, Dynisco Measurement Control, Sharon, MA; range of up to $10000 \mathrm{psi}$ ) mounted flush in the flow channel. Wall shear stresses are calculated from the pressure via a simple macroscopic balance. The displacement piston is driven by the pneumatic actuator. We chose a pneumatic system over a hydraulic system since it is more compact and amenable to transportation to a synchrotron $\mathrm{x}$-ray source.

Four threaded steel rods are coupled using nuts to the extended tie rods at the front of the pneumatic actuator ( $\mathrm{Hy}-$ droline Inc., Rockford, IL) and provide a frame to mount the reservoir block, $\mathrm{R}$. The actuator is held on an optical breadboard using edge clamps and the breadboard is mounted on a heavy table using eight $\frac{1}{4}-20$ bolts. The table is bolted to the floor of the laboratory to damp out vibrations caused by the impact when high pressures are suddenly applied across the cartridge.

\section{Pneumatic switching system}

The pneumatic actuator is driven by a tank of compressed nitrogen gas, and is controlled by a high switching speed solenoid-activated three-way spool-type valve with a closed cross-over (W7076C6331, Ross Operating Valve Co., Troy, MI). When the valve is switched, gas pressure (set by a laboratory regulator on the nitrogen tank to between 0 and $100 \mathrm{psi}$, or $0.68 \mathrm{MPa}$ ) is generated at the back of the piston in the actuator. Since rapid switching requires a large gas flow rate, which cannot be obtained from a small orifice laboratory pressure regulator, the gas from the regulator is routed to a "surge tank" mounted on the valve. This arrangement also circumvents the problem of time lag for pressure buildup due to the high compressibility of the gas. The 100:1 area ratio of the actuator can be used to generate pressures up to $68 \mathrm{MPa}$ at the end of the actuator shaft (corresponding to a wall shear stress of $\sim 0.1 \mathrm{MPa}$ ). We did not seek to achieve higher wall shear stresses since most poly- mer melts undergo slip or melt fracture beyond this value of wall shear stress. ${ }^{40,41}$ The rise and fall times for the pressure are less than $\sim 50 \mathrm{~ms}$. The solenoid valve is switched open for a controlled period of time through a computer equipped with a data acquisition board.

\section{Temperature control}

The strong dependence of isothermal crystallization kinetics on crystallization temperature motivates minimizing the spatial and temporal temperature fluctuations in the flow channel. The temperature in the heater block must be substantially higher than the melting temperature to erase melt memory of the filling process. Thereafter, the heater block must be cooled to the desired crystallization temperature and stabilized before the pressure pulse can applied. The total time for cooling should be insignificant compared to the crystallization time; it is important that the temperature not drop appreciably below $T_{\text {cryst }}$ since that could cause relatively rapid initiation of crystallization. Finally, the spatial temperature gradient between the melt reservoir (which is maintained at a temperature $>T_{M o}$ ) and the flow channel (maintained at the crystallization temperature) must be sharp.

To satisfy these requirements, the heater block has a large thermal mass, is equipped with two symmetrically mounted cartridge heaters and has channels through which a heat transfer oil can be recirculated. The cartridge heaters are regulated through a proportional integral differential (PID) feedback controller (CN76020, Omega Engineering Inc., Stamford, CT) based on the temperature of the heater block $\mathrm{H}$ as measured via a thermocouple. The heat transfer fluid (50 cs methyl silicone oil, Nye Lubricants, New Bedford, MA) is maintained at the desired crystallization temperature via a high temperature recirculating bath (model TP-12, Julabo USA, Kutztown, PA). Recirculating the heat transfer fluid through the heater block cools it relatively quickly (in $\sim 7-10 \mathrm{~min}$ ) from the melt temperature. The long thermal response time has the advantage of providing temporal stability to $\pm 0.1{ }^{\circ} \mathrm{C}$ throughout the experiment; however it limits the range of our experiments, since the quiescent crystallization time must be significantly longer than the thermal response time. The heater block is thermally isolated from the melt reservoir by means of an insulating mica "gasket" (roughly $4 \mathrm{~mm}$ thick). The melt reservoir is clad with flexible fiberglass insulation and is heated by four cartridge heaters with independent feedback PID control.

\section{E. In situ structural characterization}

A range of morphological probes to characterize the structure development and orientation in the melt is required. Observation ports are cut into the heater block and the cartridge. Flow cartridges can be equipped with appropriate windows for either optical or X-ray experiments (e.g., quartz windows $\sim 4 \mathrm{~mm}$ thick or beryllium windows $\sim 0.5 \mathrm{~mm}$ ). The windows are glued on using a high temperature epoxy (526N, Aremco Products Inc., Ossining, NY). An epoxy with a compliant nature was chosen to minimize thermal stresses induced in the windows due to the temperature changes during the experiment. 
With this arrangement, real-time optical measurements of the turbidity and birefringence (indicative of the development of crystallinity and chain orientation, respectively) of the small angle light scattering (SALS) patterns (characteristic of micron-scale lamellar superstructure) and of the wide angle $\mathrm{x}$-ray diffraction (WAXD) and small angle $\mathrm{x}$-ray scattering (SAXS) patterns (characteristic of the unit cell and nanoscale lamellar structures, respectively) can be easily accommodated into our design. For the turbidity and birefringence measurements, the optical train consists of visible laser light (red HeNe, $\lambda=632.8 \mathrm{~nm}$ ), a polarizer oriented $45^{\circ}$ to the flow direction, the sample, a polarizing beam splitting prism (10GL08AR, Newport Co., Irvine, CA), aligned $-45^{\circ}$ to the flow direction, and two photodiode detectors (PDA50, Thor Labs Inc., Newton, NJ). The intensity of light viewed through crossed polarizers, $I_{\perp}$, is measured by a photodiode on the beam transmitted through the polarizing beam splitter; the intensity of light viewed through parallel polarizers, $I_{\|}$, is recorded by a photodiode on the transverse beam. The sum of these two intensities is used to determine the total transmitted intensity, $\left(I_{\|}+I_{\perp}\right) / I_{o}$, where $I_{o}$ is the intensity of transmitted light recorded when the sample is fully amorphous. When depolarization due to light scattering is negligible, the average birefringence of the sample, $\Delta n$, may be computed from $I_{\|}$and $I_{\perp}$ using

$$
\Delta n=\frac{\lambda}{\pi d} \arcsin \sqrt{\frac{I_{\perp}}{\left(I_{\|}+I_{\perp}\right)}} .
$$

When the retardation goes over orders, the number of orders accumulated since the cessation of shear is used to determine $\Delta n$, since the initial retardation is less than one order in all our experiments.

To account for the differences in the detector gains when calculating the birefringence, a quarter wave plate with its axis along the flow direction is used for calibration at the beginning of each experiment. Care is taken to minimize the amount of stray light incident on the detectors. Neutral density filters are used to avoid saturation of the detectors. The stability of the laser source is unimportant for the birefringence measurements (since $\Delta \mathrm{n}$ involves a ratio of the intensity of light in a particular polarization state to the total intensity). However, the turbidity measurements require a stable light source. The HeNe laser used was determined to be stable to better than $2 \%$ over the timescale of our experiments which was adequate for our measurements. While the birefringence measured in our experiments is reproducible with high precision (better than 5\%) even at low values of $\Delta n\left(\Delta n \sim 10^{-3}\right)$, the accuracy of the measurements for such low birefringence is limited by the thermal stress induced birefringence in the quartz windows which is generated during heating and cooling, and by scattering of laser light off defects on the surfaces of the windows. These can be partially corrected for by subtracting the baseline value of $\Delta n$ measured at the beginning of an experiment.

Turbidity and birefringence after the onset of crystallization arise due to scattering from crystallites and are sensitive to the size, shape and anisotropy of the crystallites as well as to the difference in dielectric properties between the crystalline and amorphous regions. Thus, while turbidity is not an exact measure of the absolute degree of crystallinity, we use the rate of change of turbidity to provide a qualitative estimate of the rate of crystallization.

The $\mathrm{H}_{V}$ SALS studies use a similar setup with a polarizer at a $45^{\circ}$ angle to the flow direction in front of the sample and a $50 \mathrm{~mm}$ diam sheet polarizer crossed with respect to the first behind the sample. The scattering angle available for measurement of the SALS pattern is maximized by using a polarizer with a large diameter as the analyzer and by positioning it close to the heater block. (To prevent degradation in the quality of the dichroic polarizer due to the high temperature of the heater block, we needed to place it about 3 $\mathrm{cm}$ from H.) A two-dimensional air cooled charge coupled device (CCD) camera (Sensys KAF 0400-G0-L, Photometrics Ltd. Tucson, AZ) with antiblooming electronics was used to acquire the SALS patterns. The data are acquired with a Pentium PC at a time resolution of $2 \mathrm{~s}$.

Our instrument lends itself to easy modification to study the effects of the dynamics of orientation and relaxation of selectively deuterium labeled chains in our sample on the nature of the crystallization process by using polarization modulation infrared (IR) dichroism using an IR laser at the wavelength corresponding to the C-D stretch. The optical train used for these studies has been described previously. ${ }^{42}$ This allows us to selectively resolve the effects of a specific species (e.g., by blending deuterated high molecular weight chains into a matrix of low molecular weight polymer) on flow enhanced crystallization. Finally, the compact design enables transportation of our instrument to a synchrotron source for measurements of the SAXS and WAXD patterns in real time (we will describe the results of our SAXS and SALS studies in future publications). ${ }^{43}$

\section{RESULTS AND DISCUSSION}

We now demonstrate the features of our instrument and illustrate some of the shear-enhanced crystallization phenomena with results of selected experiments with three different isotactic polypropylenes. First, we show that the instrument imposes box-like pressure profiles while monitoring the distortion of the melt. Then, we demonstrate how simple optical measurements can be used to monitor the effect of varying shearing durations on crystallization kinetics in a relatively monodisperse polymer. Finally, we contrast the behavior of the narrow distribution material with that of a polydisperse sample and show how kinetic information can be combined with different in situ optical measurements and ex situ microscopy.

The resins used in these experiments include a polymer obtained from Aldrich, PP-250/3.7 $\left[\mathrm{M}_{w}=250 \mathrm{~kg} / \mathrm{mol}\right.$; polydispersity index $(\mathrm{PDI})=\mathrm{M}_{w} / \mathrm{M}_{n}=3.7$; melt flow index $(\mathrm{MFI})=12$ at $230^{\circ} \mathrm{C} / 2.16 \mathrm{~kg}$ load), a broad polydispersity industrial grade isotactic polypropylene from Equistar Chemical, PP-300/6 $\left(\mathrm{M}_{w} \sim 300 \mathrm{~kg} / \mathrm{mol} ;\right.$ PDI $\sim 6-8$; MFI $=12$ at $230^{\circ} \mathrm{C} / 2.16 \mathrm{~kg}$ load) also supplied by Quantum Inc., and a developmental resin with a well-defined linear architecture and relatively low polydispersity from the Dow Chemical Company, PP-186/2.1 $\quad\left(\mathrm{M}_{w}=186 \mathrm{~kg} / \mathrm{mol}\right.$; PDI $=2.1 ; \mathrm{MFI}=22$ at $230^{\circ} \mathrm{C} / 2.16 \mathrm{~kg}$ load). 


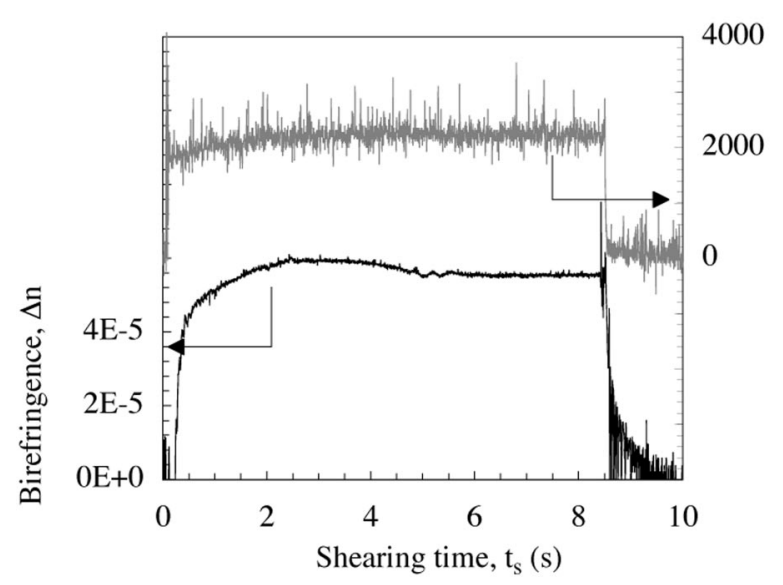

FIG. 3. Typical shearing experiment for a melt of a commercial isotactic polypropylene, PP-250/3.7, held at $200{ }^{\circ} \mathrm{C}$. The rise and fall times for the pressure are on the order of tens of ms. The birefringence shows an overshoot due to the nonlinear viscoelastic response of the melt. Data are acquired with a resolution of $5 \mathrm{~ms}$ and are presented without filtering.

\section{A. Transient melt flow}

We describe the response of molten PP-250/3.7 to an imposed deformation. The high shear stresses imposed on the polymer melt as it is driven into the slit give rise to nonlinear rheological phenomena during startup of flow which are manifested as an overshoot in the birefringence (Fig. 3). The birefringence, $\Delta n$, in the melt is related to the anisotropy in conformation of polymer chains in the melt. ${ }^{44}$ The observed value represents an average over the birefringence profile across the shear channel.

$$
\Delta n=\frac{2}{d} \int_{0}^{d / 2}\left[n_{11}(x)-n_{33}(x)\right] d x
$$

where $n_{11}-n_{33}$ is the birefringence in the plane of the flow and vorticity directions, $d$ is the optical path length and $x$ is the coordinate measured across the gap. For conditions that are not too far in the nonlinear region (viz. chain stretching that is much less than full extension), $\Delta n$ is related to the third normal stress difference, $\mathrm{N}_{3}$ by the stress optic rule.

To examine the flow behavior of the iPP melt, the reservoir and the heater block are both held at $200{ }^{\circ} \mathrm{C}$ which is well above the nominal melting point for the polymer $\left(T_{M}^{\mathrm{nom}} \sim 165^{\circ} \mathrm{C}\right)$. The pressure drop across the slit is around 2000 psi which corresponds to a wall shear stress, $\sigma_{w}$ $\sim 0.04 \mathrm{MPa}$. The pressure has sharp rise and fall times (less than $50 \mathrm{~ms}$ ), giving a well-defined shearing interval. In the case of capillary rheometers with constant piston speed, Hatzikiriakos and Dealy ${ }^{45,46}$ have shown that the buildup of the pressure transient is dominated by the compressibility of the polymer melt in the reservoir. Therefore, we checked to see if a similar effect causes the transient in the buildup of pressure in our device. However, since the pressure buildup in our experiments was independent of the amount of polymer in the reservoir, we conclude that compressibility is not an important factor in the pressure transient.

The birefringence [Eq. (1)], does not increase immediately, and shows an overshoot at around $2 \mathrm{~s}$ of shearing (Fig. 3 ). The overshoot in the time trace of the birefringence in our

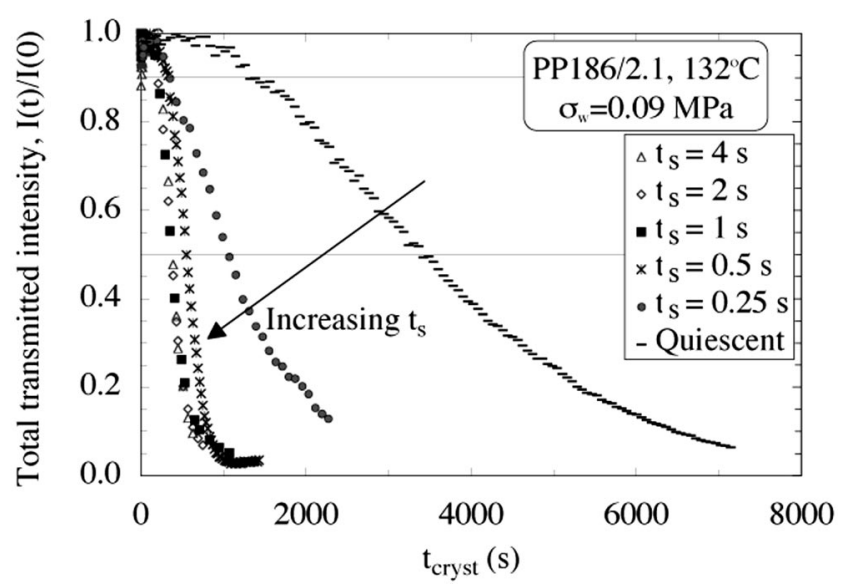

FIG. 4. Effect of shearing time on the acceleration of crystallization kinetics of PP-186/2.1. A wall shear stress of $0.09 \mathrm{MPa}$ was imposed on the relaxed, subcooled polymer melt at $132{ }^{\circ} \mathrm{C}$ and the total transmitted intensity was monitored as a function of time. Short shearing times $\left(t_{s}<1 \mathrm{~s}\right)$ dramatically decrease the time required for crystallization. Note that the shortest crystallization times $\left(t_{1 / 2} \sim 400 \mathrm{~s}\right)$ are much longer than the longest shearing time $\left(t_{s} \leqslant 4 \mathrm{~s}\right)$.

experiments is a manifestation of the overshoot in the third normal stress difference, $\mathrm{N}_{3}=\sigma_{11}-\sigma_{33}$, since the stressoptic rule holds for these flow conditions. ${ }^{44}$ Overshoots in the first normal stress difference, $\mathrm{N}_{1}$, have been observed upon startup of shear ${ }^{47,48}$ and have been rationalized in terms of the stretching of the polymer chains in the flow. Since the third normal stress difference, $\mathrm{N}_{3}$, is the sum of $\mathrm{N}_{1}$ and a relatively small quantity, the second normal stress difference, $\mathrm{N}_{2},{ }^{49}$ we expect the same physics to govern overshoots in $\mathrm{N}_{1}$ and $\mathrm{N}_{3}$. Note that the magnitude of the birefringence during shear is approximately $5 \times 10^{-5}$; values of $\Delta n$ this small are readily measured using the simple crossed polarizer arrangement.

\section{B. Crystallization of a model polymer}

We examine the effects of shearing on crystallization, starting with a low molecular weight and relatively monodisperse resin PP-186/2.1. Shearing significantly increased the rate of crystallization of PP-186/2.1 (Fig. 4); however, we have not found conditions that cause highly anisotropic growth. The experimental protocol was as indicated in Fig. 1 , allowing the polymer to relax at $240{ }^{\circ} \mathrm{C}$ for $5 \mathrm{~min}$ and then cooling to $T_{\text {cryst }}=132{ }^{\circ} \mathrm{C}$. Shearing times ranging from 250 $\mathrm{ms}$ to $4 \mathrm{~s}$ were used and the crystallization kinetics were tracked by monitoring the intensity of light transmitted through the sample. Shearing for short times (less than $1 \mathrm{~s}$ ) caused an acceleration of the crystallization kinetics as indicated by the rapid decrease in the time taken for the transmitted intensity to drop. Shearing for longer times did not show a significant further decrease in the crystallization time. These results contrast with the findings of Janeschitz-Kriegl and co-workers on a commercial Ziegler-Natta polypropylene. They observed that the crystallization time scaled with the fourth power of the wall shear rate and the second power of the shearing time $\left(t_{(\lambda / 2)} \sim \dot{\gamma}_{w}^{-4} t_{s}^{-2}\right)$. A phenomenological model was proposed ${ }^{10}$ to explain this highly nonlinear scaling behavior. Our system does not show the $t_{s}^{-2}$ scaling. We 
speculate that this might be attributed to the faster relaxation of our low molecular weight sample which might preclude the formation of the long lived "line nuclei" which form the basis of their phenomenological model. Further evidence that line nuclei are not developed comes from the absence of an increase in birefringence as the sample crystallizes, suggesting that the crystallites formed are not highly aligned.

\section{Crystallization of a commercial resin}

The more heterogeneous polypropylene, PP-300/6, shows some qualitatively different responses to shear history, including more dramatic reductions of crystallization time and highly anisotropic crystallization following sufficiently long shearing at high wall shear stresses. The polymer was melted in the reservoir at $200^{\circ} \mathrm{C}$ while the cartridge was held at $163^{\circ} \mathrm{C}$. The polymer was allowed to thermally equilibrate for $5 \mathrm{~min}$ after being extruded at a low pressure of around 500 psi to fill the subcooled slit. While "true" quiescent crystallization at this low subcooling would take place on the order of days, the polymer melt even in nominally "quiescent" conditions in this experiment crystallizes in a few hours due to the considerable preshearing experienced during the low pressure extrusion to fill the flow channel. $^{50}$ After thermal equilibration, extrusion was resumed at a pressure drop of 2600 psi ( wall shear stress $=0.05 \mathrm{MPa}$ ) for $4 \mathrm{~s}$, and the crystallization behavior monitored over a period, $t_{\text {cryst }}$ of $2000 \mathrm{~s}$.

Solidification following this treatment produces a very different microstructure in the material near the walls than in the center of the channel. Near the surface [Fig. 5(a)], a highly oriented, densely nucleated structure is observed. At greater depth, the anisotropy decreases abruptly, but the density of the nuclei is still high relative to the quiescently crystallized material. At the core, the structure is spherulitic. This spatial variation in structure correlates qualitatively with the depth dependent deformation history. The shear rate is highest near the wall [Fig. 5(b)], as is the total strain applied to the material. The deformation rate decreases with position moving toward the center of the slit. At the center, the material is undeformed (the precise velocity profile depends on the rheological properties of the specific material). The shear thinning behavior of the present sample leads to a velocity profile [Fig. 5(b)] that is blunted relative to the Poiseuille flow associated with Newtonian fluids.

In real time we do not have access to depth-selective measurements of the structure in the material. Instead, we use information on the final structure to interpret measured quantities that average over the gap. Ex situ analysis of the crystallized sample using optical and electron microscopies as well as X-ray scattering helps us better understand how different structures contribute to the turbidity, birefringence, SALS etc. and provide a foundation for interpreting real-time depth averaged observations. For example, in interpreting the birefringence observed during flow, it is important to recognize that the signal is dominated by a relatively thin region near each wall, due to the nonlinear dependence of the normal stresses on shear rate. Flow-induced changes in the rate of increase in the turbidity of the samples are also dominated (a)

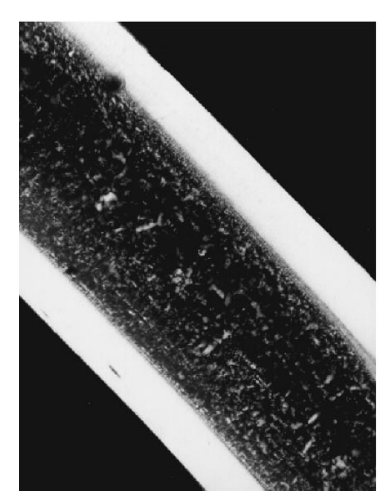

(b)

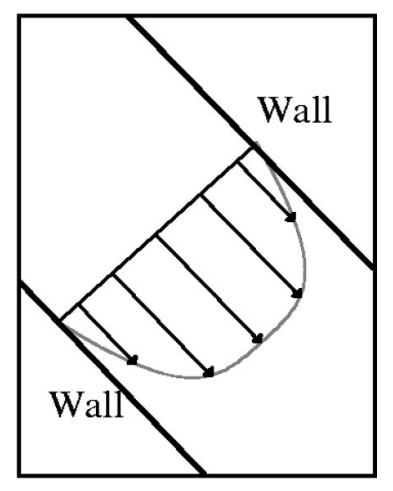

FIG. 5. (a) Ex situ polarized light micrograph of the cross section (width $=0.5 \mathrm{~mm}$ ) of PP300/6 after shearing at a wall shear stress of 0.05 $\mathrm{MPa}$ for $4 \mathrm{~s}$. The sample strip runs from the upper left to the lower right, between the black regions in the lower left and the upper right. The bright regions on each side of this strip have a high birefringence oriented along the flow direction indicative of highly oriented crystallites. The region near the middle of the strip contains spherulites. Thus, shearing has induced formation of a pronounced skin-core morphology. The scattering from the skin dominates the turbidity and birefringence observed in the in situ optical measurements. (b) Schematic of the relatively blunt velocity profile expected for a shear-thinning polymer melt under pressure driven flow. The velocity profile was calculated by fitting rheological data for PP300/6 to a power law constitutive equation. A no-slip boundary condition was assumed to hold at the walls of the die.

by the material near the walls, since the polymer at the center continues to follow quiescent crystallization kinetics. With this spatial heterogeneity in mind, we turn to interpretation of the time resolved measurements of birefringence and turbidity.

Shearing for $4 \mathrm{~s}$ accelerated the crystallization kinetics by several orders of magnitude and the transmitted intensity fell to half of its initial value in just over $500 \mathrm{~s}$ (Fig. 6). The birefringence during the pressure pulse (Fig. 6, inset) showed the development of an oriented structure which did not fully relax after cessation of shear. This structure was observed only for shearing times greater than $2 \mathrm{~s}$. For shorter shear durations, no such oriented structure was formed during flow and the crystallites formed subsequently were not oriented on a macroscopic length scale. Strongly oriented crystalline structures (as indicated by the behavior of $I_{\perp}$ ) were formed only when the melt birefringence showed the long lived feature (Fig. 6). Thus the formation of the structure in the melt during shearing correlates with the subsequent growth of crystalline structures oriented on macroscopic length scales. While this increase in birefringence during shearing has been observed by previous researchers, ${ }^{51}$ its relationship to subsequent anisotropic structure had not been shown. This clearly demonstrates the need to measure the melt distortion and to use it to interpret the nature of the crystallization process.

\section{Application to other materials and flow geometries}

The results described here in Sec. IV focus exclusively on our investigations into the relation between melt flow history and microstructure formation in semicrystalline polymers. However, the small sample requirement of our instrument also opens up studies of model polymers or other materials available only in quantities of grams, and makes it 


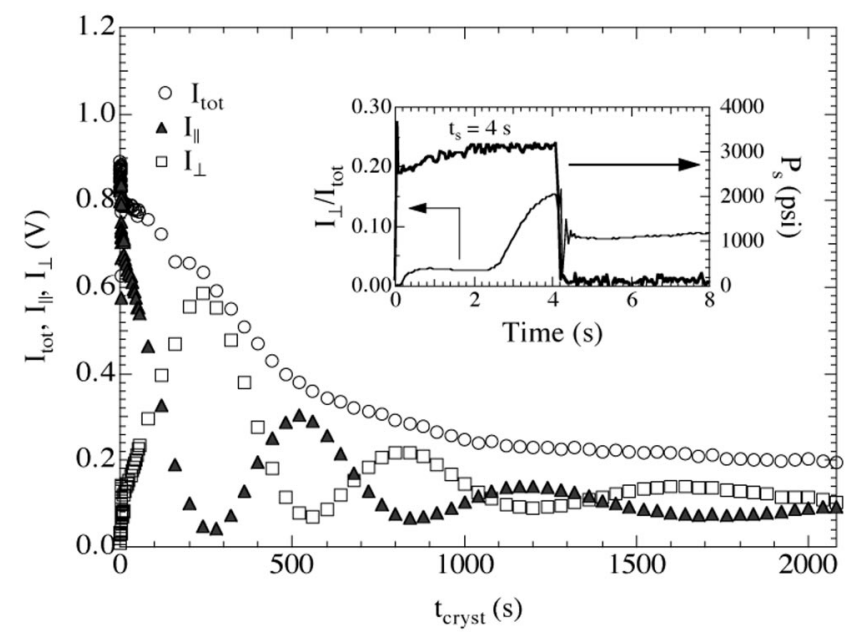

FIG. 6. Crystallization of a polydisperse isotactic polypropylene, PP300/6, after shearing at a wall shear stress of $0.05 \mathrm{MPa}$ for $4 \mathrm{~s}$. The deformation of the polymer melt during the pressure pulse is monitored (inset). The unusual increase in the birefringence starting at $2.5 \mathrm{~s}$ indicates formation of an oriented structure, which does not relax after shear cessation. This is seen to correlate with the formation of anisotropic crystallites.

possible to study their behavior under commercial processing-like conditions. The unique ability to incorporate a range of structural probes (with data collection at millisecond time resolution) makes it a valuable tool for the investigation of processing induced structure in a variety of systems such as liquid crystalline polymers, blends and block copolymers. Finally, while we are interested in using simple flow geometries to unravel the physics of flow-enhanced crystallization, the versatile design makes it easy to use complicated flow geometries and impose arbitrary flow fields to investigate processing-structure-property relations in materials under more realistic processing-like conditions.

\section{ACKNOWLEDGMENTS}

The authors would like to acknowledge financial support from Procter \& Gamble and the Schlinger fund that made this project possible. One of the authors (G.K.) would like to acknowledge support from a Landau fellowship. The authors are very grateful to Dr. A. Prasad (Equistar Chemical) for providing the commercial grade polypropylene, PP8004MR (PP-300/6), and to Dr. R. L. Sammler and Dr. C. P. Bosnyak (The Dow Chemical Company) for supplying the narrow polydispersity isotactic polypropylene (Dow developmental resin, PP-186/2.1). Dr. R. L. Sammler also gave a critical reading of the manuscript and very useful comments. Dr. G. Smedley and Professor R. C. Flagan (Caltech) gave valuable advice regarding the design of the instrument.

${ }^{1}$ Resins Report, Mod. Plastics 75, 74 (1998).

${ }^{2}$ D. C. Bassett, Principles of Polymer Morphology (Cambridge University Press, New York, 1981).

${ }^{3}$ D. W. van Krevelen, Chimia 32, 279 (1978).

${ }^{4}$ M. Fujiyama and T. Wakino, J. Appl. Polym. Sci. 43, 57 (1991).

${ }^{5}$ A. Peterlin, Colloid Polym. Sci. 265, 357 (1987).

${ }^{6}$ L. H. Wang and R. S. Porter, J. Polym. Sci., Part B: Polym. Phys. 22, 1645 (1984).

${ }^{7}$ I. Arvanitoyannis, E. Tsatsaroni, E. Psomiadou, and J. M. V. Blanshard, J. Appl. Polym. Sci. 51, 1753 (1994).
${ }^{8}$ L. C. Lopez, R. C. Cieslinski, C. L. Putzig, and R. D. Wesson, Polymer 36, 2331 (1995).

${ }^{9}$ Y. Ulcer, M. Cakmak, J. Miao, and C. M. Hsiung, J. Appl. Polym. Sci. 60, 669 (1996).

${ }^{10}$ S. Liedauer, G. Eder, H. Janeschitz-Kriegl, P. Jerschow, W. Geymayer, and E. Ingolic, Int. Polym. Process. 8, 236 (1993).

${ }^{11}$ S. Liedauer, G. Eder, and H. Janeschitz-Kriegl, Int. Polym. Process. 10, 243 (1995).

${ }^{12}$ P. Jerschow and H. Janeschitz-Kriegl, Rheol. Acta 35, 127 (1996).

${ }^{13}$ P. Jerschow and H. Janeschitz-Kriegl, Int. Polym. Process. 12, 72 (1997).

${ }^{14}$ A. J. McHugh and B. Khomani, Int. Polym. Process. 5, 252 (1990).

${ }^{15}$ A. J. McHugh, D. A. Tree, B. Pornnimit, and G. W. Ehrenstein, Int. Polym. Process. 6, 208 (1991).

${ }^{16}$ Y. Ulcer and M. Cakmak, Polymer 38, 2907 (1997).

${ }^{17}$ R. Phillips, G. Herbert, J. News, and M. Wolkowicz, Polym. Eng. Sci. 34, 1731 (1994).

${ }^{18}$ H. Ito, K. Minagawa, J. Takimoto, K. Tada, and K. Koyama, Int. Polym. Process. 11, 363 (1996).

${ }^{19}$ C. M. Hsiung, M. Cakmak, and J. L. White, Polym. Eng. Sci. 30, 967 (1990).

${ }^{20}$ H. X. Huang, J. Appl. Polym. Sci. 67, 2111 (1998).

${ }^{21}$ G. Kalay and M. J. Bevis, J. Polym. Sci., Part B: Polym. Phys. 35, 265 (1997).

${ }^{22}$ M. Saiu, V. Brucato, S. Piccarolo, and G. Titomanlio, Int. Polym. Process. 7, 267 (1992).

${ }^{23}$ T. H. Yu and G. L. Wilkes, J. Rheol. 40, 1079 (1996).

${ }^{24}$ J. R. Dees and J. E. Spruiell, J. Appl. Polym. Sci. 18, 1053 (1974).

${ }^{25}$ R. K. Gupta and K. F. Auyeung, Polym. Eng. Sci. 29, 1147 (1989).

${ }^{26}$ S. Misra, F. M. Lu, J. E. Spruiell, and G. C. Richeson, J. Appl. Polym. Sci. 56, 1761 (1995).

${ }^{27}$ M. Cakmak and J. C. Kim, J. Appl. Polym. Sci. 64, 729 (1997).

${ }^{28}$ T. W. Haas and B. Maxwell, Polym. Eng. Sci. 9, 225 (1969).

${ }^{29}$ K. Kobayashi and T. Nagasawa, J. Macromol. Sci., Phys. B4, 331 (1970).

${ }^{30}$ A. Wereta, Jr. and C. G. Gogos, Polym. Eng. Sci. 11, 19 (1971).

${ }^{31}$ R. R. Lagasse and B. Maxwell, Polym. Eng. Sci. 16, 189 (1976).

${ }^{32}$ P. G. Andersen and S. H. Carr, Polym. Eng. Sci. 16, 217 (1976).

${ }^{33}$ P. G. Andersen and S. H. Carr, Polym. Eng. Sci. 18, 215 (1978).

${ }^{34}$ R. D. Ulrich and F. P. Price, J. Appl. Polym. Sci. 20, 1077 (1976).

${ }^{35}$ C. H. Sherwood, F. P. Price, and R. S. Stein, J. Polym. Sci., Polym. Symp. 63, 77 (1978).

${ }^{36}$ M. D. Wolkowicz, J. Polym. Sci., Polym. Symp. 63, 365 (1978).

${ }^{37}$ C. Tribout, B. Monasse, and J. M. Haudin, Colloid Polym. Sci. 274, 197 (1996).

${ }^{38}$ S. Vleeshouwers and H. E. H. Meijer, Rheol. Acta 35, 391 (1996).

${ }^{39}$ A. E. Hamielec and J. B. P. Soares, Prog. Polym. Sci. 21, 651 (1996).

${ }^{40}$ C. W. Macosko, Rheology: Principles, Measurements and Applications (VCH, Wertheim, 1993).

${ }^{41}$ R. G. Larson, Rheol. Acta 31, 213 (1992).

${ }^{42}$ R. M. Kannan and J. A. Kornfield, J. Rheol. 38, 1127 (1994).

${ }^{43}$ K. Guruswamy, R. K. Verma, J. A. Kornfield, F. Yeh, and B. S. Hsiao (unpublished).

${ }^{44} \mathrm{H}$. Janeschitz-Kriegl, Polymer Melt Rheology and Flow Birefringence (Springer, Berlin, 1983).

${ }^{45}$ S. G. Hatzikiriakos and J. M. Dealy, Polym. Eng. Sci. 34, 493 (1994).

${ }^{46}$ J. M. Dealy, Rheol. Acta 34, 115 (1995).

${ }^{47}$ E. V. Menezes and W. W. Graessley, J. Polym. Sci., Part B: Polym. Phys. 20, 1817 (1982)

${ }^{48}$ D. S. Pearson, A. D. Kiss, L. J. Fetters, and M. Doi, J. Rheol. 33, 517 (1989).

${ }^{49}$ R. G. Larson, Constitutive Equations for Polymer Melts and Solutions (Butterworths, London, 1988).

${ }^{50}$ Linear viscoelastic data for PP-300/6 indicates that the time for relaxation of stresses in the melt at $\mathrm{T}_{\text {cryst }}$ is lesser than the time allowed for thermal equilibration. However, in our experiments, the memory of the high strain distortion experienced by the melt during the initial low pressure extrusion is retained and it decreases the crystallization time.

${ }^{51}$ P. Jerschow, Ph.D. thesis, Johannes Kepler University, Linz, Austria, 1994. 